\title{
SELECCIÓN DE PROGENITORES DE MAÍZ PARA LA OBTENCIÓN DE SEMILLA HÍBRIDA POR PEQUEÑOS AGRICULTORES 1
}

\author{
Roberto Valdivia ${ }^{2}$, Víctor A. Vidal², Mauro Sierra ${ }^{2}$
}

\begin{abstract}
RESUMEN
Selección de progenitores de maíz para la obtención de semilla híbrida por pequeños agricultores. El costo creciente de semilla híbrida de maíz ha limitado la productividad del cultivo en México. Por eso, los agricultores tienden a sembrar híbridos de segunda generación para reducir costos. Una mejor opción es utilizar el modelo Productor-Experimentador en la decisión de sembrar semilla de cruzas intervarietales. El objetivo del estudio fue describir la experiencia que ha tenido un grupo de productores de Santa María del Oro, Nayarit, en la producción, utilización y mejoramiento de cruzas intervarietales de maíz. En 1996, los productores sembraron seis experimentos donde observaron que la cruza $\mathrm{B} 840 \mathrm{xC} 343$ rindió mejor que nueve híbridos comerciales. Con esta motivación, en 1997/98, los agricultores produjeron 20 toneladas de semilla de ocho cruzas. Los rendimientos de semilla fueron de 2 a $3 \mathrm{t} / \mathrm{ha}$, así la semilla fue distribuida en la comunidad a menos del 50\% del costo del híbrido comercial. En 1998, se sembraron alrededor de 400 ha con su semilla producida y se evaluaron las ocho cruzas, en cuatro experimentos. Los resultados mostraron que las mejores cruzas fueron P3028xA7573 y C385xD880. La primera cruza mostró una heterosis promedio y sobre el mejor progenitor de 4,9\% y $4.1 \%$, respectivamente; mientras que la segunda, tuvo similarmente una heterosis del $30 \%$ sobre un progenitor. A partir de 1999, los progenitores de la mejor cruza se mejorarán mediante un sistema de selección recurrente recíproca de medios hermanos, dos años por ciclo.
\end{abstract}

\begin{abstract}
Maize progenitor selection to obtain hybrid seeds by small farmers. The seed of actual high yielding hybrids are very expensive and local farmers tend to use advanced generations to reduce costs. A better choice is to produce seed of maize intervarietal hybrids. The objective of this research was to describe the experience of a group of maize producers in the seed production, utilization and improvement of their parents of intervarietal hybrids. The study was conducted in Nayarit State, Mexico. In 1996, a group farmers, supported by a technical researcher, evaluated two maize intervarietal crosses. The cross $\mathrm{B} 840 \mathrm{xC} 343$ yielded better than nine commercial hybrids. This motived the farmers to produce 20 tons of seed in eight intervarietal crosses during the winter season of 1997/98. The cost of the seed production was 50\% less than the cost of commercial hybrid seed. The eight intervarietal crosses were commercial planted in about 400 hectars and were evaluated in four experiments by the farmers. The results showed that two crosses, P3028xA7573 and $\mathrm{C} 385 \mathrm{xD} 880$, had the best results. The first cross had a heterosis of $4.1 \%$ over the high yielding parent and the second a heterosis of 30\% over a parent. Since 1999, the parents of the best intervarietal cross is being improved by reciprocal recurrent selection of half siblings, two years per cycle.
\end{abstract}

\section{INTRODUCCIÓN}

En el estado de Nayarit, México, se siembran alrededor de 60,000 ha con semilla de maíz de origen mejorado. La semilla mejorada es predominantemente híbrida de alto rendimiento con una tendencia a ser cada vez de cruza trilineal o simple. Sin embargo, sólo el 50 $\%$ de dicha superficie se siembra con semilla mejorada lo que indica que se está usando semilla de híbridos de segunda generación y criollos con la pérdida del $20 \%$ de la producción por el uso de esa semilla (Valdivia y Vidal, 1995). Esto obedece a que los agricultores han observado que sus niveles de productividad, relación beneficio-costo, se han reducido, a pesar de obtener rendimientos unitarios de cuatro a siete $\mathrm{t} / \mathrm{ha}$ con el uso de semilla híbrida de calidad. Se considera que el costo creciente de la semilla híbrida y la estabilización del precio internacional del grano de maíz son las principales razones de la caída de la productividad del cultivo.

Para superar la crisis de la productividad del cultivo de maíz se requiere el desarrollo de un nuevo modelo de producción agrícola. Donde la participación directa del productor es importante. Los principales

\footnotetext{
1 Presentado en la XLV Reunión anual del PCCMCA, Guatemala, 1999.

2 Instituto Nacional de Investigaciones Forestales, Agrícolas y Pecuarias (INIFAP). Apartado Postal 100; CP 63300; Santiago Ixcuintla, Nayarit, México
} 
componentes tecnológicos que se deben investigar y desarrollar un sistema de labranza cero, así como la producción y uso de la fertilización orgánica (Palacios, 1997) y semilla propia. Este programa de investigación y desarrollo se debe implementar con el modelo del Productor-Experimentador (Mendoza et al, 1998; Valdivia y Villarreal, 1998). La semilla que pueden producir los mismos agricultores es un tipo de cruza intervarietal entre variedades mejoradas de amplio uso por los agricultores y que de preferencia deberían ser los mejores híbridos comerciales. La bondad de rendimiento y adaptación de las cruzas intervarietales se ha reconocido en diversos estudios que se han realizado en diferentes regiones y con diverso germoplasma mejorado (Mena, 1985; Guzmán et al, 1992; González et al, 1993; Villanueva et al, 1994; Valdivia et al, 1999).

El objetivo de esta investigación fue la de describir la experiencia que han tenido un grupo de productores de maíz en la producción de semilla, utilización y mejoramiento de progenitores de híbridos intervarietales de maíz.

\section{MATERIALES Y MÉTODOS}

\section{Modelo productor-experimentador}

El modelo Productor-Experimentador se basa en una relación afectiva entre un técnico investigador y un grupo de 16 productores que representan una comunidad, que asegura que los cambios tecnológicos se hagan con bases fundamentadas. Es un modelo de investigación en la acción, con características tanto de educación como de investigación, que permite al agricultor tomar conciencia de cada elemento de su proceso de producción, cuestionar los logros obtenidos o detectar las fallas para encontrar soluciones por sí mismo. El modelo Productor-Experimentador se basa en el método general del tecnólogo que involucra un proceso de entender y explicar; así como de dar soluciones a las ineficiencias técnicas mediante la conducción de experimentos, previa formulación de hipótesis (Palacios, 1997).

\section{Desarrollo de la investigación}

El estudio se realizó en los ejidos de Buckingham y San José de Mojarras, pertenecientes al municipio de Santa María del Oro, Nayarit, México, que cubre una superficie de 13,000 ha de maíz. Es una región con clima tropical con transición de subtropical; altitud de 0 a $1,200 \mathrm{msnm}$

En 1996 se inició el programa con la participación de 16 productores en cada ejido. De ellos, seis productores: Florencio, Jesús, Mariano, Cándido, José y Anto- nio participan en el desarrollo de la semilla propia. Las siembras de maíz se realizan, en el verano, mayormente bajo condiciones de buen temporal donde la lluvia supera los de $800 \mathrm{~mm}$ anuales; y en el ciclo de invierno, se realiza la producción de semilla bajo condiciones de riego, donde el agua se conduce por el suelo. Esta parte del programa se inició en 1996 con la evaluación de dos cruzas intervarietales en comparación a 10 híbridos comerciales de mayor siembra en la comunidad. La evaluación se hizo en seis experimentos de los que se les llama de "genotipo por ambiente" donde cada agricultor se hace responsables de un experimento que lo siembra dentro de su predio. Las parcela la constituye cuando menos cuatro surcos a todo lo largo de su predio. Los datos y mediciones lo hacen en los surcos centrales y en cada parcela realizan tres muestreos al azar de cosecha que reemplazan a las repeticiones. Las significancias estadísticas se consideran al $10 \%$. El técnico les enseña a tomar los datos en forma conjunta y en los años siguientes lo hace sólo el mismo agricultor. El manejo de datos lo hace el técnico.

Los resultados de los experimentos de 1996 motivaron a los agricultores a producir semilla de cruzas intervarietales en el invierno de 1997/98. Se produjo la semilla de ocho cruzas diferentes en los ejidos de Buckingham, San José de Mojarras y Miguel Hidalgo (Cuadro 1). Cada cruza se hizo en lotes aislados con una proporción de 3:1 de surcos hembra y macho. En la producción de la semilla, los agricultores fueron asesorados por el técnico investigador y un despacho particular de técnico. El financiamiento fue otorgado por una caja de ahorro de solidaridad que es formada y manejada por los mismos agricultores del municipio.

En 1998/98, la semilla obtenida fue sometida a un control de calidad mediante la siembra de alrededor de 400 ha comerciales dentro de Santa María del Oro y simultáneamente se sembraron tres experimentos tipo genotipo por ambiente en San José de Mojarras y cinco en Buckingham. Con base a estos resultados, los agricultores seleccionarán las cruzas que más les convenga, de acuerdo a su calidad productiva de grano y semilla. Los progenitores de las cruzas seleccionadas se someterán a un proceso de mejoramiento genético mediante el sistema de selección recurrente recíproca de medios hermanos que requiere tres siembras que se tienen que realizar en dos años. El método no necesita control artificial de la polinización, esto es, bolsas para polinizar. Al completar el primer ciclo de selección, los agricultores serán dueños de su propia semilla.

\section{RESULTADOS Y DISCUSIÓN}

En la Figura 1, se muestran los resultados, promedio de los seis experimentos, de la evaluación de dos 
Cuadro 1. Progenitores de híbridos comerciales utilizados en la producción de semilla híbrida. OI-1997/98.

\begin{tabular}{lllr}
\hline Nombre de la cruza & Progenitores de las cruzas & \multicolumn{1}{c}{ Ejido } & Superficie (ha) \\
\hline 1. HD-1203 & HV313XD880 & Buckingham & 2,0 \\
2. CFD-1265 & C385F2XD880 & Buckingham & 1,0 \\
3. PD-3908 & P3028XD880 & Buckingham & 0,5 \\
4. AD-8453 & A7573XD880 & Buckingham & 0,5 \\
5. DP-3895 & D867XP3028 & Miguel Hidalgo & 1,0 \\
6. CD-1265 & C385XD880 & S.J. de Mojarras & 1,0 \\
7. PA-10601 & P3028XA7573 & S.J. de Mojarras & 1,0 \\
8. CA-75C & CiclónXa7573 & S.J. de Mojarras & 1,0 \\
\hline
\end{tabular}

cruzas intervarietales realizada en 1996. El mejor genotipo resultó ser el híbrido comercial A7573, con un rendimiento de 7,5 t/ha. Sin embargo, la cruza intervarietal $\mathrm{B} 840 \mathrm{xC} 343$, produjo 7,3 t/ha y superó al resto de genotipos que incluyeron los híbridos comerciales H430, H431, A7597, P3288, P3066, Tornado, Ciclón, C385 y C220. La otra cruza, B840xA7559, rindió 6,7 t/ha con lo que se comportó similar a la mayoría del resto de genotipos híbridos comerciales. Con estos resultados, los agricultores expresaron su entusiasmo en la posibilidad de producir semilla de cruzas intervarietales que habían personalmente observado de suficiente buena calidad de producción. Por otro lado, las cruzas que evaluaron no fueron necesariamente las mejores, las cuales fueron C343xA7579 y C343xH430 (Valdivia et al, 1999).

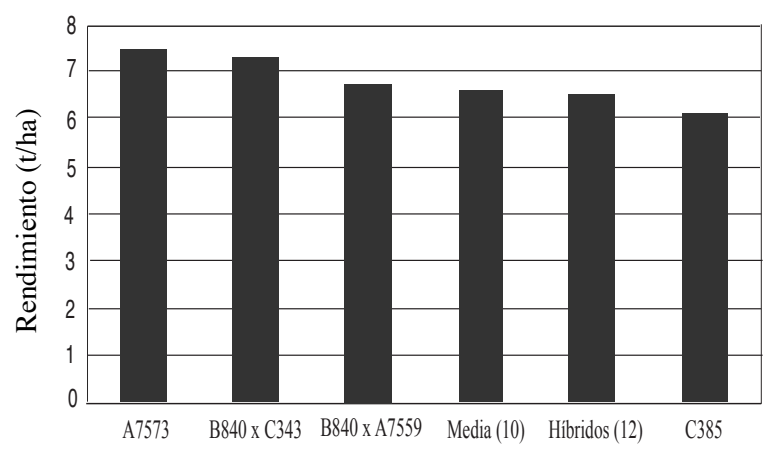

Figura 1. Comportamiento de cruzas de maíz en comparación a híbridos comerciales

\section{Producción de semilla}

Los agricultores lograron producir $20 \mathrm{t}$ de semilla de las ocho cruzas intervarietales (Cuadro 1). El costo promedio de producción de semilla fue de $\$ 20,000$ pesos por hectárea. Se tuvieron rendimientos de semilla de 2 a $3 \mathrm{t} / \mathrm{ha}$; el costo por $\mathrm{kg}$ de semilla fue de $\$ 6,67 \mathrm{y}$ $\$ 10,0$ pesos, respectivamente. Los productores de semilla distribuyeron en su comunidad entre \$200 y \$ 250 pesos por saco de semilla suficiente para sembrar una hectárea. Esto es, a un $50 \%$ del costo de la semilla híbrida comercial. Beneficios agregados fueron la disponibilidad local de la semilla y el dinero de los jornales quedó en la misma comunidad.

\section{Control de calidad}

En la siembra comercial de 400 ha que se hizo en la región se obtuvieron resultados satisfactorios a todos los agricultores que sembraron las cruzas intervarietales, lo cual fue avalado en diferentes demostraciones que realizaron agricultores para agricultores.

Por otro lado, los resultados de la evaluación de cuatro experimentos llevados a cabo por los agricultores de Buckingham y los tres de San José de Mojarras, se presentan en los Cuadros 2 y 3, respectivamente. En Buckingham, las cruzas tuvieron una heterosis promedio y sobre el mejor progenitor de 42,1 y $32,0 \%$ sobre sus progenitores híbridos comerciales. Sobresalieron las cruzas PA10601 (P3028xA7573) y CD1265 (C385xD880) con rendimientos superiores a las 6,2 t/ha y heterosis positiva; superaron a los híbridos comerciales A7573 y P3028, los cuales tienen amplia aceptación en la región y se vendieron entre $\$ 550$ y \$ 600 pesos por saco.

En San José de Mojarras, promedio de los tres experimentos, las cruzas tuvieron una heterosis positiva del 12,9 \% sobre el promedio del mejor progenitor (Cuadro 3). Tres cruzas tuvieron rendimiento superior a las 6 t/ha, AD8453 (A7573XD880), PD3908 (P3028xD880) y PA10601 (P3028xA7573). Superaron a los mejores testigos híbridos comerciales, P3028 y A7573. Se observa la consistencia de la cruzaPA10601 que es formada por los mejores híbridos de la región, A7573 y P3028.

Los resultados confirman la favorable respuesta que han demostrado diferentes tipos de cruzas intervarietales valoradas en diferentes regiones maiceras de México (Mena, 1985; Guzmán et al, 1992; González et al, 1993; Villanueva et al, 1994; Valdivia et al, 1999).

En este estudio resalta el hecho de que los mismos agricultores han participado en la producción de semi- 
Cuadro 2. Rendimiento y heterosis de híbridos intervarietales de maíz evaluados en Buckingham, Nay. PV-1998/98.

\begin{tabular}{rllllccrr}
\hline & Genotipo & Antonio & José & Lucio & Mariano & Media & HPP $(\%)$ & HPS (\%) \\
\hline 1 & PA10601 & 5639 & 6384 & 6599 & 7000 & 6405 & 4.9 & 4,1 \\
2 & CD1265 & 5049 & 7285 & 5760 & 6776 & 6218 & 30,0 & 30,0 \\
3 & A7573 (T) & 6074 & 6787 & 6339 & 5469 & 6167 & 0,0 & 0,0 \\
4 & DP3895 & 5527 & 7491 & 6516 & 4991 & 6131 & 2,0 & 1,3 \\
5 & P3028 (T) & 4764 & 7480 & 6202 & 5756 & 6050 & 0,0 & 0,0 \\
6 & D867 (T) & 5065 & 7872 & 5402 & 5532 & 5968 & 0,0 & 0,0 \\
7 & CFD1265 & 6433 & 4491 & 5852 & 6488 & 5816 & 21,6 & 21,6 \\
8 & AD8453 & 4984 & 6536 & 5456 & & 5659 & 3,3 & 0,6 \\
9 & CA75C & 4916 & 5666 & 5812 & 6105 & 5625 & $-8,8$ & $-8,8$ \\
10 & Opaco & 4777 & 6005 & 5491 & 4508 & 5195 & 0,0 & 0,0 \\
11 & PD3908 & 3456 & 5527 & 5808 & & 4930 & $-9,0$ & $-12,9$ \\
12 & D880 (T) & 4903 & 4421 & 4959 & 4851 & 4784 & 0,0 & 0,0 \\
13 & HD1203 & 4802 & 4331 & 4711 & 4536 & 4595 & $-1,9$ & $-3,9$ \\
14 & HV313 (T) & 3719 & 4374 & 5192 & 5037 & 4580 & 0 & 0 \\
& Media & 5008 & 6046 & 5721 & 5587 & 5591 & 42,1 & 32,0 \\
\hline
\end{tabular}

$\mathrm{HPP}=$ Heterosis promedio progenitor medio; HPS= Heterosis progenitor superior

$\mathrm{T}=$ Testigo Híbrido Comercial

lla y valoración de las cruzas y personalmente se han convencido de la calidad de sus cruzas intervarietales, particularmente se han dado cuenta que buenos progenitores híbridos comerciales producen las mejores cruzas intervarietales. Aparentemente esto obedece a la divergencia genética que tienen los híbridos comerciales en virtud a la calidad de selección a que han sido sometidos (Sánchez et al, 1973).

\section{Mejoramiento de progenitores de cruzas interva- rietales}

El esquema de mejoramiento de los progenitores de la cruza intervarietal que se seleccione, se presenta en la Figura 2. Los dos progenitores se les identifica como población A y población B. Estas se llevan a una población segregante en F2. Se siembran por separado y en lotes aislados y se cosechan 1000 mazorcas provenientes de las mejores 1000 plantas. Se seleccionan las mejores 500 mazorcas y en el siguiente ciclo de invierno se siembran las 500 mazorcas en surcos por mazorca, intercalados por surcos macho de la otra población.
Se desespigan las hembras para ser polinizadas por la otra población; se cosecha semilla de los mejores 100 surcos hembra. En el verano se evalúan las 100 cruzas de prueba A.F2xB.F2 y las B.F2xA.F2. En invierno se recombinan las mejores 20 progenies de cada población con semilla remanente A.F2 y B.F2. Se repite el ciclo con la cosecha de la mazorca con semilla A.F3 y B.F3 de las mejores 1000 plantas.

En este esquema se necesita que los productores hagan las selecciones, los hijos de los productores tomen los datos y el técnico diseñe, dirija y maneje los datos. También es posible incorporar las características de calidad de proteína a las poblaciones, pero se requieren al menos tres retrocruzas para la incorporación y el apoyo de un laboratorio para seleccionar los granos con las características QPM modificados a vítreo.

Se hipotetiza que las cruzas A.F2x B.F2 pueden reducir su rendimiento en un $15 \%$ en comparación con las cruzas similares, pero en $\mathrm{F} 1 \mathrm{xF} 1$. Ese $15 \%$, se puede recuperar en el primer ciclo de selección.

Cuadro 3. Rendimiento y heterosis de híbridos intervarietales de maíz evaluados en San José de Mojarras, Nay. PV-1998/98.

\begin{tabular}{rlccccrr}
\hline & Genotipo & Francisco & Pablo & Daniel & Media & HPP(\%) & HPS (\%) \\
\hline 1 & AD8453 & 5323 & 5426 & 8077 & 6276 & 17,5 & 16,3 \\
2 & PD3908 & 4910 & 5634 & 8044 & 6196 & 8,3 & 2,4 \\
3 & PA10601 & 5362 & & 6934 & 6148 & 2,1 & 1,6 \\
4 & P3028 (TC) & 5552 & 4861 & 7735 & 6050 & 0,0 & 0,0 \\
5 & DP3895 & 4155 & 7050 & 6792 & 5999 & $-0,8$ & $-0,8$ \\
6 & CFD1265 & 4637 & 5037 & 7036 & 5570 & 5,4 & 5,4 \\
7 & A7573 (TC) & 5190 & 3727 & 7270 & 5396 & 0,0 & 0,0 \\
8 & D880 (TC) & 5716 & 3366 & 6769 & 5284 & 0,0 & 0,0 \\
9 & HV313 (TC) & 4427 & 4692 & 5969 & 5029 & 0,0 & 0,0 \\
10 & CD1265 & 4465 & 3449 & 7062 & 4992 & $-5,5$ & $-5,5$ \\
11 & HD1203 & 5995 & 4064 & 4758 & 4939 & $-4,2$ & $-6,5$ \\
12 & Opaco & 4211 & 2786 & 6699 & 4566 & 0,0 & 0,0 \\
13 & CA75C & 4371 & 3082 & 5472 & 4309 & $?$ & $?$ \\
& Media & 4947 & 4431 & 6817 & 5398 & 22,7 & 12,9 \\
\hline
\end{tabular}




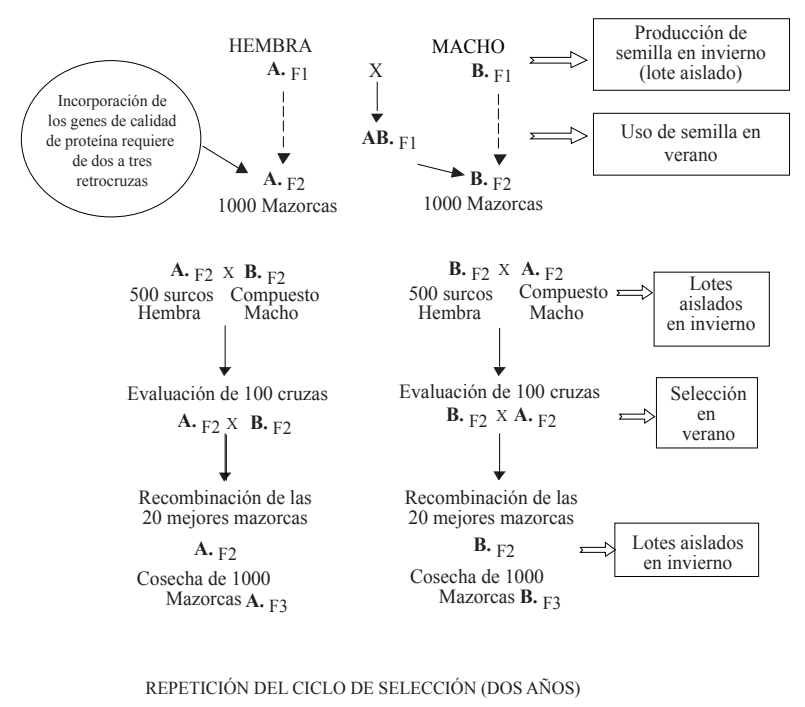

Figura 2. Esquema del mejoramiento de semilla de cruzas intervarietales de maíz mediante selección recurrente recíproca de medios hermanos.

\section{CONCLUSIONES}

El modelo productor-experimentador está funcionando en lograr un proceso de mejoramiento continuo de la productividad del maíz. El uso de semilla de cruzas intervarietales es la mejor opción para reducir costos de cultivo manteniendo la misma producción y que puede mejorar en la medida que se mejoren las cruzas.

Los agricultores participaron directamente, con el apoyo del técnico investigador, en la producción de semilla de híbridos intervarietales, someterlos a un proceso de control de calidad que verificó la calidad productiva de las cruzas, así como la buena producción de su semilla. La cruza intervarietal que puede utilizarse para mejorarse genéticamente es la que forman los híbridos comerciales P3028xA7573. Esta cruza produce mejor que cualquiera de sus progenitores y su semilla se produce a costos que representan el $50 \%$ del costo de la semilla híbrida comercial de esos progenitores.

\section{LITERATURA CITADA}

GONZÁLEZ S., C.; RON P., J.; RAMÍREZ D, J. L. 1993. Cruzas entre híbridos comerciales de maíz. Rev. Fitotec. Mex. 16: 30-41.

GUZMÁN M., L., PALACIOS C., V.; RON P., J. 1992. Cruzas intervarietales en el cultivo de maíz (Zea mays L.) con la participación de productores en la región de Nextipac, Municipio de Zapopan, Jalisco. Memoria XIV Congreso Nacional de Fitogenética. p. 292.

MENA M., S. 1985. Formación de cruzas intervarietales de F2 híbridos. In: Memorias XI demostración agrícola y pecuaria. Departamento de Filotecnia. Facultad de Agricultura. Universidad de Guadalajara.

MENDOZA R., J. L.; MACIAS C., J; VILLARREAL F, E.; QUIJANO C., J.A; PAREDES M., R. 1998. Aplicación de un método de diagnóstico para la inducción de un cambio tecnológico en el cultivo de maíz en el norte de Sinaloa. Folleto Técnico Núm. 16. Campo Experimental Valle del Fuerte del Instituto Nacional de Investigaciones Forestales, Agrícolas y Pecuarias (INIFAP), México. 32 p.

PALACIOS C., V. 1997. Fertilizante orgánico. Tecnología para una rentabilidad sostenible. Publicación especial. Instituto Nacional de Investigaciones Forestales, Agrícolas y Pecuarias (INIFAP). Convenio INIFAP-MASECA. México. 24 p.

SÁNCHEZ M., R.; MOLÍAN G., J; CASAS D., E. 1973. Efecto de dosis de germoplasma exótico y de citoplasma tropical sobre el rendimiento de cruzas tropicales x Mesa Central en maíz (Zea mays L.). Colegio de Postgraduados, Chapingo, México. Agrociencia 11:151-179.

VALDIVIA B., R.; VIDAL M, V. A. 1995. Efecto de generaciones avanzadas en la producción de diferentes tipos de híbridos de maíz. Rev. Fitotec. Mex. 18: 69-76.

VALDIVIA B., R.; VILLARREAL F., E. 1998. Metodología de diagnóstico técnico en el cultivo del maíz. In: Memorias del XVII Congreso de Fitogenética. Acapulco, Gro. México. p. 273

VALDIVIA B., R.; VIDAL M., V. A.; SIERRA M., M. 1999. Heterosis en cruzas intervarietales de maíz. In: Memorias XIV reunión anual del Programa Cooperativo Centroamericano de Mejoramiento de Cultivos y Animales (PCCMCA), Guatemala. p.42 